\title{
Análise fatorial de sintomas depressivos e ocorrência de quedas em idosos
}

\author{
Factor analysis of depressive symptoms and occurrence of falls among the elderly
}

\author{
Amanda Gilvani Cordeiro Matias ${ }^{1} \bowtie$, Marília de Andrade Fonseca ${ }^{2}$, Marcos Antônio Almeida Matos ${ }^{3}$ \\ 1 Fisioterapeuta e bióloga. Mestre em Meio Ambiente e Saúde. Doutoranda em Medicina e Saúde pela Escola Bahiana de Medicina e Saúde Pública. \\ Docente na Universidade Federal da Bahia - Instituto Multidisciplinar em Saúde (UFBA-IMS). Vitória da Conquista, BA. \\ 2 Fisioterapeuta. Mestre em Ciências da Saúde. Doutoranda em Medicina e Saúde pela Escola Bahiana de Medicina e Saúde Pública. \\ Docente na Universidade Estadual do Sudoeste da Bahia (UESB). Jequié, BA. \\ ${ }_{3}^{3}$ Médico. Mestre e Doutor em Ortopedia e Traumatologia pela Universidade de São Paulo. Docente no Programa de Pós-Graduação Stricto Sensu \\ da Escola Bahiana de Medicina e Saúde Pública. Salvador, BA.
}

\section{RESUMO}

Objetivos: Determinar a prevalência de sintomas depressivos e correlacionar a ocorrência destes com o autorrelato de quedas em idosos, utilizando a estrutura fatorial da Escala de Depressão Geriátrica de Yesavage-15 (EDG-15).

Métodos: Estudo de corte transversal realizado de julho a outubro de 2014. Participaram idosos voluntários, vinculados ao Centro de Convivência do Idoso na cidade de Vitória da Conquista, Bahia, que foram selecionados por amostragem não probabilística e por conveniência e responderam a um questionário à EDG-15, após serem informados sobre a pesquisa e assinarem o termo de consentimento livre e esclarecido. Para verificar a correlação bivariada entre sintomas depressivos e autorrelato de quedas, utilizou-se o teste de Pearson. Foi aplicada estatística multivariada através da técnica de análise fatorial, que verificou a inter-relação dos itens da EDG-15, visando à redução do número de variáveis em fatores explicativos.

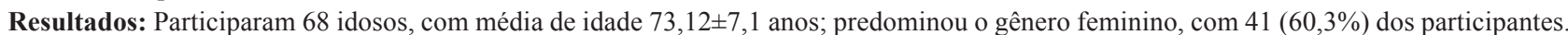
Foram detectados sintomas depressivos em 35 (51.4\%) dos idosos, sendo 23 (33.8\%) mulheres. A queda foi autorrelatada por 33 idosos $(48,5 \%)$. Houve correlação positiva e significativa entre o autorrelato de queda e sintomas depressivos nos participantes $(r=0,383, p<0,001)$ A consistência interna da EDG-15 foi avaliada pelo teste de Alpha de Cronbach, obtendo valor aceitável $(0,73)$; o teste que verificou a aplicabilidade da escala foi o Kaiser-Meyer-Olkin, cujo coeficiente obtido $(0,658)$ demonstrou a adequação para o método de análise fatorial. Após a aplicação da análise fatorial, destacaram-se dois fatores que agruparam oito itens extraídos da escala, capazes de explicar $68 \%$ da variância total da EDG-15.

Conclusões: A prevalência da depressão foi relevante na amostra estudada. A maior carga fatorial foi representada nas dimensões de ansiedade, anedonia, medo, isolamento e apatia. A correlação entre autorrelato de quedas e sintomas depressivos foi significativa. Os dados apontam para a necessidade de maior atenção à saúde mental do idoso e enfatizam a importância de intervenções precoces de rastreamento dos sintomas depressivos visando à adoção de medidas preventivas.

DECRITORES: idoso fragilizado; transtorno depressivo; acidentes por quedas.

\section{ABSTRACT}

Aims: To determine the prevalence of depressive symptoms and correlate them with self-reported falls among the elderly using the Yesavage-15 Geriatric Depression Scale (GDS-15).

Methods: A cross-sectional study was conducted from July to October 2014. Elderly volunteers from the Community Center for the Elderly located in Vitória da Conquista, State of Bahia, Brazil, participated in the study. They were selected by non-probability convenience sampling and answered the GDS-15 questionnaire after being informed about the study objectives and signing a free consent form.

Results: A total of 68 elderly individuals with a mean age of 73.12 \pm 7.1 years were included in the study, and female participants accounted for $60.3 \%$ of the sample. Depressive symptoms were detected in $35(51 \%)$ individuals, among which $23(33.8 \%)$ were female. Falls were self-reported by 33 elderly individuals $(48.5 \%)$. There was a positive and significant correlation $(r=0.383$, $p<0.001)$ between self-reported falls and depressive symptoms among the participants. The internal consistency of the GDS-15 was assessed by Cronbach's alpha, which yielded an acceptable value (0.73). The Kaiser-Meyer-Olkin measure of sampling adequacy was used to verify the applicability of the GDS-15, yielding a value of 0.658 , which means the scale was adequate for the factor analysis method. Two factors, into which eight items were grouped, stood out after the factor analysis and were able to explain $68 \%$ of the total variance of the GDS- 15 .

Conclusions: The prevalence of depression was relevant. The dimensions of anxiety, anhedonia, fear, isolation, and apathy showed the largest factor loads. The correlation between self-reported falls and depressive symptoms was significant. The data indicate it is necessary to pay special attention to the mental health of the elderly and also highlight the importance of the early detection of depressive symptoms, thus allowing for preventive measures.

KEY WORDS: frail elderly; depressive disorder; accidental falls. 


\section{INTRODUÇÃO}

O envelhecimento biológico ocasiona uma série de alterações morfofuncionais e psicocognitivas, sendo proeminentes as doenças de cunho psiquiátrico, entre as quais a depressão aparece como uma das mais comuns entre idosos. Conjetura-se sua associação à maior morbimortalidade, ao aumento do risco de quedas e à demanda por serviços de saúde [1].

A doença depressiva relaciona-se com fatores biológicos e psicossociais, gerando alterações físicas, funcionais e incapacitantes que interferem na capacidade funcional e comportamental, desencadeando perturbações somáticas e do humor no indivíduo acometido. Esses distúrbios acarretam mudanças no perfil epidemiológico, aumentando a frequência das incapacidades que comprometem o desempenho e a qualidade de vida do idoso $[1,2]$.

Segundo o Instituto Brasileiro de Geografia e Estatística, no censo de 2010 a população brasileira era de 190.732 .694 pessoas, sendo que de 10 a $11 \%$ estavam acima de 60 anos, com expectativa de vida de 78 anos para mulheres e 72 anos para homens. Esta tendência à inversão no modelo de crescimento populacional, na qual ocorre o progressivo aumento de idosos e redução de jovens, é um fenômeno mundial de destacado impacto $[3,4]$.

A participação das quedas em idosos na mortalidade proporcional por causas externas no Brasil, entre 1984 a 1994, cresceu de 3\% para 4,5\%. Uma pesquisa epidemiológica realizada com idosos na cidade de São Paulo apontou prevalência de quedas de $30 \%$, as recorrentes representando $11 \%$ entre idosos institucionalizados. Os mesmos autores relatam que em outros países esta prevalência pode alcançar $60-75 \%$ e destacam que no Brasil ainda são escassos os estudos sobre a prevalência de quedas em idosos [5].

Os episódios de queda predominam como a principal causa de morte acidental em idosos. Cerca de $40-60 \%$ destas ocorrências levam a alguma lesão; 30 $50 \%$ são de menor gravidade, $5-6 \%$ são injúrias graves e 5\% exibem fraturas. Estes fatos chamam a atenção para os riscos na população idosa, demandando mais pesquisas em busca de evidências sobre os índices de quedas, provavelmente ainda subestimados [6].

A depressão em idosos encontra-se associada a aumento da morbimortalidade, limitação da funcionalidade, negligência no autocuidado, incapacidades e risco de quedas. Essas condições, quando associadas ao uso de medicamentos, agregam maiores fragilidades à saúde do idoso [7].
A depressão e o risco de queda são condições comuns que geram prejuízos à saúde e à integridade do idoso. A presença de depressão como um dos fatores preditores para o aumento do risco de quedas foi investigada por Birderman et al. [8] por meio de um estudo de coorte que utilizou a Escala de Depressão Geriátrica de Yesavage (EDG) com 15 itens (EDG-15) aplicada a 283 idosos, dos quais $12 \%$ referiram quedas frequentes em um ano e $25,5 \%$ tiveram triagem positiva para sintomas depressivos. Os pesquisadores mostraram que essas variáveis coexistem na medicina geriátrica e recomendam a EGD-15 como escore útil para discriminar os sintomas depressivos que são mais importantes como fator de risco para episódios de queda. A queda é uma condição prevenível, simples de rastrear e sensível a ações preventivas direcionadas [8].

Pessoas de todas as faixas etárias apresentam risco de cair, entretanto essas ocorrências são mais prevalentes em idosos, podendo ocasionar fraturas, incapacidades, longos períodos de hospitalização e morte. Além de sobrecarregar os sistemas assistenciais, aumentam os custos da assistência de média e alta complexidade $[9,10]$. Várias situações associadas às quedas estão sendo estudadas, como envelhecimento, vertigens, desânimo, uso de psicotrópicos e sintomas depressivos [7,9,11]. A identificação dos fatores associados à queda pode contribuir para esclarecimento das suas causas e possibilitar a inserção de medidas preventivas nesse grupo populacional $[4,12]$.

Conhecer as condições de saúde dos idosos revestese de grande importância científica e social, visto que esses transtornos afetam a capacidade funcional, apresentam elevada frequência e comprometem negativamente a qualidade de vida dos indivíduos [13]. O uso sistemático de escalas validadas, de boa confiabilidade avaliativa sobre a depressão, pode viabilizar a detecção precoce desse distúrbio na prática clínica e assistencial. A EDG-15 é uma escala simples que serve a esse propósito $[13,14]$.

O objetivo deste estudo foi determinar a prevalência de sintomas depressivos, correlacionar esses sintomas ao autorrelato de quedas em idosos vinculados a um centro de convivência de terceira idade e verificar a estrutura fatorial da EDG-15.

\section{MÉTODOS}

Foi realizado um estudo exploratório com idosos vinculados ao Centro de Convivência dos Idosos (CCI) da Prefeitura Municipal de Vitória da Conquista, no sudoeste da Bahia. Os critérios de inclusão foram idosos autônomos, não institucionalizados, que 
voluntariamente aceitaram participar da pesquisa e não apresentaram impedimento para responder ao questionário e realizar o teste da caminhada. A amostra não probabilística foi composta por conveniência, recrutando, de forma consecutiva, idosos que frequentavam o centro de convivência todas as quartas-feiras para as atividades de educação em saúde coordenadas pela equipe local.

O convite para o idoso participar da pesquisa ocorreu através do termo de consentimento livre e esclarecido. A cada voluntário foram explicados o objetivo e os benefícios da pesquisa. O Comitê de Ética em Pesquisa da Faculdade Independente do Nordeste (FAINOR) aprovou a realização da pesquisa através do parecer consubstanciado $\mathrm{n}$ - 759.548/14.

Aos que aceitaram participar e assinaram o termo de consentimento foi aplicado um questionário elaborado pelos autores, com informações sociodemográficas, acrescido da pergunta: "Caiu nos últimos 12 meses? Sim/não". Para coletar informações referentes aos sintomas de depressão, foi utilizada a EDG-15. Foi adotado o ponto de corte de $\geq 6$ para identificar a presença significativa dos sintomas de depressão [15].

A EDG é uma escala validada para triagem da depressão em idosos, em dois formatos: com 30 itens (versão longa) e com 15 itens (versão curta). A forma longa usa um ponto de corte de 11 pontos e a versão curta adota um ponto de corte de seis pontos. A EDG é de uso gratuito, sendo disponível em vários idiomas. Pesquisas de validade da escala curta, a EDG15 , demonstraram pequenas diferenças referentes ao ponto de corte mais adequado, no mínimo de 5/6 como indicativo de depressão [1,6]. A EDG-15 avalia os sintomas do transtorno depressivo nas duas últimas semanas antes da entrevista, através de 15 itens que pesquisam humor deprimido, anedonia (perder o prazer, ou desânimo para fazer as coisas), insônia ou hipersônia, cansaço ou letargia, mudança no apetite ou peso, sentimento de culpa ou inutilidade, dificuldade com concentração e memória, lentidão ou inquietação e ideias suicidas $[11,16,17]$.

Os resultados da EDG-15 foram dicotomizados para facilitar a análise fatorial e compor as dimensões que versam a escala; para determinar os dois níveis depressivos, adotou-se como corte $5 / 6$, ou seja, até cinco pontos "sem depressão" e a partir de seis pontos "presença de depressão". Também foram categorizados em depressão leve $\leq 10$ e depressão severa $\geq 11[17,18]$.

Os dados foram analisados utilizando o pacote estatístico IBM-SPSS versão 20.0. O teste de Kolmogorov-Sminorv verificou a normalidade dos dados. Para verificar a correlação bivariada entre sintomas depressivos e autorrelato de quedas, utilizou-se o teste de Pearson. Foi considerada a significância de $\mathrm{p}<0,05$ e o intervalo de confiança (IC) usado foi de 95\%. Utilizou-se a estatística multivariada através da técnica de análise fatorial que verificou a inter-relação dos itens da EDG-15, visando à redução do número de variáveis em fatores explicativos. Foi utilizado o teste de Kaiser-Meyer-Olkin para verificar a adequação da amostra para análise fatorial. A consistência interna da escala foi testada pelo cálculo Alfa de Cronbach. A análise fatorial visa reduzir em quantidade os itens da escala e destacar os mais relevantes. Ao verificar a estrutura e inter-relações de variáveis observadas, a análise fatorial define o agrupamento dos fatores que melhor explicam as covariâncias da escala $[11,19]$.

\section{RESULTADOS}

Neste estudo foram incluídos 68 idosos, com média de idade de $73 \pm 7$ anos, entre os quais $41(60,3 \%)$ eram do gênero feminino. A idade variou de 61-88 anos. Da amostra observada, $33(48,5 \%)$ relataram ter caído nos últimos doze meses. Foi verificada a normalidade dos dados, cuja significância foi de $p<0,001$. Quanto ao nível de escolaridade, houve maior frequência de idosos com escolarização. Na Tabela 1 estão apresentadas as características da amostra estudada.

Tabela 1. Características sociodemográficas da amostra e ocorrência de quedas em idosos na cidade de Vitória da Conquista, Bahia, Brasil, 2014.

\begin{tabular}{|c|c|c|}
\hline Variáveis & Categoria & $\mathrm{N}(\%)$ \\
\hline Grupo etário (anos) & $\begin{array}{l}60-70 \\
71-80 \\
81-90\end{array}$ & $\begin{array}{ll}28 & (41.1) \\
29 & (42.6) \\
11 & (16.3)\end{array}$ \\
\hline Sexo & $\begin{array}{l}\text { Feminino } \\
\text { Masculino }\end{array}$ & $\begin{array}{ll}41 & (60.3) \\
27 & (39.7)\end{array}$ \\
\hline Estado civil & $\begin{array}{l}\text { Em união } \\
\text { Sem união }\end{array}$ & $\begin{array}{ll}30 & (44.1) \\
38 & (55.9)\end{array}$ \\
\hline Escolaridade & $\begin{array}{l}\text { Não escolarizado } \\
\text { Escolarizado }\end{array}$ & $\begin{array}{ll}26 & (38.2) \\
42 & (61.8)\end{array}$ \\
\hline Risco de depressão & $\begin{array}{l}\text { Sem depressão } \\
\text { Depressão } \\
\text { Depressão grave }\end{array}$ & $\begin{array}{ll}32 & (47.1) \\
30 & (44.1) \\
05 & (7.4)\end{array}$ \\
\hline $\begin{array}{l}\text { Autorrelato de queda nos últimos } \\
12 \text { meses }\end{array}$ & $\begin{array}{l}\text { Não } \\
\text { Sim uma vez } \\
\text { Sim duas vezes } \\
\text { Sim três vezes } \\
\text { Sim quatro vezes }\end{array}$ & $\begin{array}{ll}35 & (51.5) \\
20 & (29.4) \\
10 & (14.7) \\
02 & (2.9) \\
01 & (1.5)\end{array}$ \\
\hline
\end{tabular}

Foram rastreados com sintomas depressivos 35 $(51.5 \%)$ idosos que obtiveram mais de seis pontos no escore utilizado (Tabela 1). A correlação de Pearson 
Tabela 2. Frequência dos sintomas depressivos dos itens da Escala de Depressão Geriátrica de Yesavage-15 em idosos, na cidade de Vitória da Conquista, Bahia, Brasil, 2014.

\begin{tabular}{|c|c|c|c|}
\hline Itens da Escala & Média & Desvio padrão & $\mathbf{N}(\%)$ \\
\hline Está satisfeito(a) com a vida? & 18 &, 384 & $12(17.6)$ \\
\hline Diminuiu parte de suas atividades e interesses? & ,68 & 471 & $46(67.6)$ \\
\hline Sente que a vida está vazia? &, 40 & ,493 & $27(39.7)$ \\
\hline Aborrece-se com frequência? & ,35 &, 481 & $24(35.3)$ \\
\hline Sente-se de bem com a vida maior parte do tempo? &, 37 & ,486 & $25(36.8)$ \\
\hline Teme que algo ruim possa acontecer? &, 31 &, 465 & $21(30.9)$ \\
\hline Sente-se feliz a maior parte do tempo? &, 38 &, 490 & $26(38.2)$ \\
\hline Sente-se frequentemente desamparado(a)? &, 22 & ,418 & $15(22.1)$ \\
\hline Prefere ficar em casa a sair e fazer coisas novas? &, 54 &, 502 & $37(54.4)$ \\
\hline Acha que tem mais problemas de memória que outros &, 18 &, 384 & $12(17.6)$ \\
\hline Acha que é maravilhoso estar vivo agora? &, 13 &, 341 & $09(13.2)$ \\
\hline Vale a pena viver como vive agora? &, 26 &, 444 & $18(26.5)$ \\
\hline Sente-se cheio(a) de energia? &, 54 &, 502 & $37(54.4)$ \\
\hline Acha que sua situação tem solução? &, 16 &, 371 & $11(16.2)$ \\
\hline Acha que tem muita gente em situação melhor? & ,74 & ,444 & $50(73.5)$ \\
\hline
\end{tabular}

foi fraca, mas positiva entre o nível de depressão identificada e o número de quedas autorrelatadas $(\mathrm{r}=0.383$ e valor de $\mathrm{p}<0.001)$.

A escala utilizada neste estudo adotou ponto de corte em $5 / 6$ que definiu não caso/caso por item. $\mathrm{Na}$ Tabela 2 está expressa a análise descritiva com as respostas do percentual e frequência dos sintomas indicativos de depressão pelos itens da EDG-15.

A etapa inicial da análise fatorial consistiu no teste de verificação da confiabilidade do total da escala, que obteve coeficiente aceitável de 0,73 do Alfa de Cronbach, sendo este coeficiente considerado adequado para prosseguir a análise fatorial. Outro teste que verificou a aplicabilidade da análise fatorial foi o Kaiser-Meyer-Olkin, cujo coeficiente foi 0,658 , atestando a adequação para o método em questão. A hipótese nula na qual não há correlação entre as variáveis foi verificada pelo teste de esfericidade de Bartlett, que obteve significância de $p<0,001$, inferindo que a matriz da escala é apropriada para a extração de fatores, ou seja, para exploração e demonstração da estrutura de variáveis inter-relacionadas.

A análise fatorial visa reduzir ao mínimo possível o número de fatores com o máximo de variância, através da segunda etapa da metodologia de extração que é a análise dos componentes principais, a qual gera uma matriz de comunalidades e um gráfico específico. As comunalidades são correlações de cada variável explicada pelos fatores: quanto maior a comunalidade, maior o seu poder de explicação. Usualmente o valor mínimo aceitável de poder explicativo do fator é de 0,5 . Valores menores são extraídos por decisão do programa estatístico. Na Tabela 3 estão descritos os fatores já reduzidos e suas correlações após a análise dos componentes principais (comunalidades) que destacou os itens proeminentes da EDG-15 (2, 3, 4, 7, $9,13,14$ e 15 ) com porcentagem maior que $50 \%$. Na etapa seguinte a análise dos componentes principais transformou as correlações dos fatores, que foram reduzidos para dois grupos independentes, retendo apenas valores superiores a 1 , denominados autovalor (eigenvalues) com percentual de 68,432\% que explica a variabilidade total acumulada dos itens.

Tabela 3. Correlações reduzidas dos itens da Escala de Depressão Geriátrica de Yesavage-15 aplicada a idosos, em Vitória da Conquista, Bahia, Brasil, 2014.

\begin{tabular}{|c|c|}
\hline Comunalidades - variâncias das correlações & Extração (\%) \\
\hline 2. Diminuiu parte de suas atividades e interesses? & ,763 \\
\hline 3. Sente que a vida está vazia? &, 674 \\
\hline 4. Aborrece-se com frequência? & ,795 \\
\hline 7. Sente-se feliz a maior parte do tempo? & ,703 \\
\hline 9. Prefere ficar em casa a sair e fazer coisas novas? & ,809 \\
\hline 12. Vale a pena viver como vive agora? & ,714 \\
\hline 13. Sente-se cheio(a) de energia? &, 787 \\
\hline Acha que tem muita gente em situação melhor? & ,759 \\
\hline
\end{tabular}


A Figura 1 representa a última ponderação da análise dos componentes principais depois de reduzir e agrupar em apenas dois fatores a variância total dos itens que mais se relacionam. Assim, no eixo $\mathrm{Y}$ do gráfico encontra-se o Fator $1=3,8294$ e no eixo $\mathrm{X}$ o Fator $2=1,7767$, representando a carga fatorial dos eigenvalues ajustados pelo programa. Os fatores são compostos por duas ou mais variáveis/itens da escala avaliada, que são determinadas pela etapa seguinte do método, denominada de rotação varimax, que decide as variáveis latentes que irão compor cada um dos dois fatores, mantendo excluídos os itens com carga fatorial baixa, $\leq 5$. Uma mesma variável não pode compor fatores distintos [19]. Assim, na Tabela 4 estão apresentados os componentes extraídos

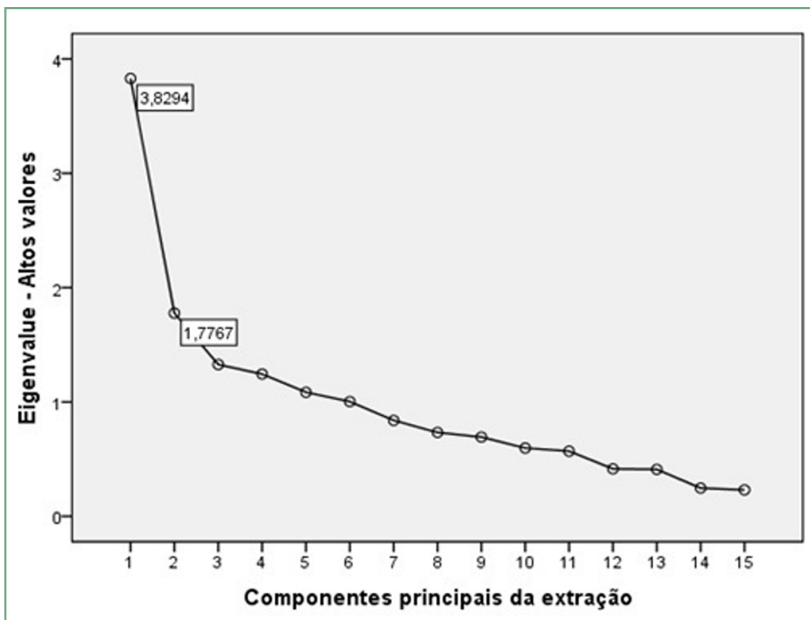

Fator $1=3,8294 \%$ e Fator $2=1,7767 \%$ explicam dos eigenvalues ajustados pelo programa para os dois fatores.

Figura 1. Variância dos componentes principais da Escala de Depressão Geriátrica de Yesavage-15 avaliados em idosos na cidade de Vitória da Conquista, Bahia, Brasil, 2014.

Tabela 4. Matriz de cargas fatoriais dos componentes principais após redução em dois fatores dos itens da Escala de Depressão Geriátrica de Yesavage-15 em idosos na cidade de Vitória da Conquista, Bahia, Brasil, 2014.

\begin{tabular}{l|c}
\hline F1 $-\mathbf{E V}=\mathbf{3 , 8 2 9 4}$ Estado de Humor Desanimo/insatisfação & Cargas \\
\hline 07. Sente-se feliz a maior parte do tempo & 0.629 \\
11. Acha que é maravilhoso estar vivo agora & 0.756 \\
12. Vale a pena viver como vive agora & 0.625 \\
13. Sente-se cheio(a) de energia & 0.768 \\
15. Sente que tem muita gente em situação melhor & 0.617 \\
\hline F2-EV $=\mathbf{1 , 7 7 6 7}$ Sinais cognitivos e neurovegetativos & Cargas \\
\hline 02. Diminuiu parte de suas atividades e interesses & 0.689 \\
06. Teme que algo ruim possa acontecer & 0.647 \\
09. Prefere ficar em casa a sair e fazer coisas novas & 0.682 \\
\hline
\end{tabular}

F1: Fator 1 - Dimensão do estado de humor

F2: Fator 2 - Dimensão cognitiva e neurovegetativa

$\mathrm{EV}$ : eingenvalue (autovalor) da matriz rotacionada varimax, que neste estudo reduziu a dois fatores ponderados pelas cargas fatoriais e interpretação descritiva de seus conteúdos.

A matriz de cargas fatoriais do método varimax minimizou os itens ajustados, agrupando-os nos dois fatores da seguinte maneira: o Fator 1, composto por cinco itens da escala $(7,11,12,13$ e 15) e o Fator 2, com três itens (2, 6 e 9). Assim, de acordo com o método de análise fatorial após rotação varimax, esses itens da escala foram os que mais influenciaram na determinação da depressão através da EDG-15, aplicada à amostra deste estudo.

Os itens da escala agrupados no Fator 1 podem ser interpretados numa dimensão do estado de humor com quadro depressivo através da tristeza, anedonia, desânimo, não se sentir feliz ou cheio de energia no dia-a-dia, interferindo na satisfação ou contentamento do respondente. Nesse agrupamento com maior carga fatorial destacam-se os itens 7 e 12 da EDG-15 ("sentir-se feliz a maior parte do tempo" e "valer a pena viver como vive agora"), referindo-se ao estar ou não satisfeito com a vida por algum motivo.

Os itens agrupados no Fator 2 representam as dimensões relacionadas aos sinais cognitivos e neurovegetativos que interferem e comprometem as atividades da vida diária, condições presentes na depressão que podem interferir na capacidade funcional, e por consequência, influenciar a queda. $\mathrm{O}$ item do Fator 2 com maior carga fatorial foi o número 2, seguido do item 9 da escala ("diminuiu parte de suas atividades e interesses"), os quais refletem condição cognitiva e somática (apatia, medo e inatividade e desânimo) que são característicos no quadro depressivo, conforme agrupamento apresentado na Tabela 4.

\section{DISCUSSÃO}

Os resultados das características sociodemográficas deste estudo foram semelhantes às tendências descritas na literatura $[11,20]$ no que se refere à dimensão psicopatológica depressiva. Estes resultados foram consistentes entre os idosos, demonstrados pelas correlações existentes entre medo, desânimo e irritabilidade, e o comprometimento importante do humor, peculiar na condição depressiva.

Houve predominância do sexo feminino no autorrelatado de quedas e no rastreamento positivo dos sintomas depressivos; estas informações são compatíveis com os resultados de pesquisas realizadas com idosos $[1,21]$. A depressão com predominância no gênero feminino foi pesquisada entre 150 idosos cujos 
resultados obtiveram uma prevalência do episódio depressivo em $1,9 \%$ dos homens e 3,2\% das mulheres [16].

A característica da amostra referente ao estado civil, que mostrou prevalência de idosos sem união conjugal (separados, viúvos ou que nunca se casaram) foi semelhante aos resultados encontrados por Pelegrin et al. [18]. Os dados apontam para a prevalência do sexo feminino e para o fato de que as mulheres vivem mais do que os homens.

A depressão é uma condição clínica que vem se tornando frequente em idosos. Estudos epidemiológicos indicam taxas de prevalência de 1 a $16 \%$ de depressão em idosos que vivem na comunidade, sendo que naqueles que apresentam doenças preexistentes os índices são maiores, até $45 \%$. Esses estudos respaldaram a EDG-15 como um dos instrumentos mais utilizados para o rastreamento da depressão entre adultos jovens e idosos, sendo uma escala com medidas válidas e confiáveis para triagem do transtorno depressivo $[4,12]$.

Situações vivenciadas pelos idosos, como viuvez, solidão, dependência, doenças e apatias de diversas ordens, podem contribuir para desestruturações psíquicas que condicionam o quadro de depressão, causando um dos mais importantes danos à saúde e à qualidade de vida do idoso. A depressão não diagnosticada e não tratada piora a condição funcional e aumenta o risco para outras doenças e para episódios de quedas [2,21]. Estes pesquisadores apontam para a necessidade de pesquisas com instrumentação simples e confiável para rastreio da depressão. A EDG utilizada por esses autores que aplicaram a mesma técnica de análise fatorial obtiveram boa consistência interna, conforme o índice de Cronbach e os fatores latentes identificados que foram semelhantes ao presente estudo [21].

A pesquisa de Cwikel \& Ritchie (1990) citada por Paradela et al. [22], realizada em Jerusalém, adotando ponto de corte $5 / 6$, demonstrou especificidade de $57 \%$ e sensibilidade de $72 \%$. Paradela et al. [22] citam ainda a pesquisa de Lyness et al., realizada em 1998 em ambulatórios de Nova Iorque, nos Estados Unidos, com ponto de corte $5 / 6$, que mostrou uma sensibilidade de $92 \%$ e especificidade de $81 \%$; a de Fountoulakis et al. em 1999 , que usou o ponto de corte $6 / 7$, encontrando $92,2 \%$ de sensibilidade e $95,2 \%$ de especificidade, sendo que a consistência interna medida pelo alfa de Cronbach obteve coeficiente de 0,94; e a de Lim et al. em 2000, que adotou ponto corte de $4 / 5$ ao estudar idosos chineses, relatando boa adequação do ponto de corte, com sensibilidade de $84 \%$ e especificidade de $85,7 \%$ na curva ROC [22].
Estudo que aplicou a EDG-15 a 64 idosos em ambulatório psiquiátrico no Brasil, com o objetivo de rastrear a depressão, adotou ponto de corte $5 / 6$, obtendo sensibilidade de $85,4 \%$ e especificidade de $73,9 \%$. Quando o ponto de corte foi mudado para $6 / 7$ pontos, os autores obtiveram sensibilidade de $84,8 \%$ e especificidade de $67,7 \%$, concluindo que a escala mostrou-se confiável e válida em ambos os pontos de corte, quando aplicada a pacientes de ambulatório especializado [23].

Neste estudo, receberam destaque as novas variáveis geradas pelo método análise dos componentes principais, que expressaram a capacidade de informação contida nos itens da escala aplicada. Este método fatorial reduziu a dimensionalidade dos itens e destacou aqueles mais representativos e correlacionados, que foram: medo de momentos futuros, anedonia que compromete a capacidade de sentir prazer na vida, ansiedade e condição apática ligada ao isolamento social. Esses itens são semelhantes aos encontrados na literatura consultada $[11,13,24]$, apontando que tais condições são determinantes para o risco depressivo, como observado o item 2 da escala: "diminuiu parte de suas atividades de interesse", que obteve maior pontuação na matriz de comunalidade, sugerindo forte influência para deflagrar o risco de depressão.

O aumento da prevalência de depressão em idosos desperta preocupação, estimulando pesquisas na última década $[1,4,11]$. As pesquisas epidemiológicas encontram grandes variações na prevalência da depressão em idosos, com índices que oscilam entre $13,4 \%$ e $49 \%$ [14,30]. Em um estudo de coorte elaborado a partir de uma clínica de atendimento primário com 283 idosos, 25,5\% apresentaram depressão leve e $46,7 \%$ depressão acentuada, entre os quais 33,3\% apresentavam depressão grave [8]. No presente estudo os percentuais foram semelhantes, mesmo frente à diversidade que envolve o envelhecimento populacional no Brasil. Chama a atenção para a prevalência da depressão que é três a cinco vezes maior na população idosa do que em adultos jovens, com o agravante de ser uma doença ainda subdiagnosticada [17].

Os altos índices de depressão em idosos observados nas recentes publicações refletem a importância do diagnóstico e tratamento precoce e oportuno, pois o envelhecimento impõe naturalmente limitações ao desempenho funcional $[16,17]$. Os dois fatores com carga mais expressiva nesta pesquisa destacaram as dimensões latentes do estado de humor e sinais cognitivos (anedonia, desmotivação, medo, inatividade) como indícios do estado depressivo, 
fatores estes que explicam $68 \%$ da variância total pela análise dos componentes principais, somando-se ao percentual de episódios de queda autorrelatados e correlacionados. Esses dados são semelhantes aos de outras pesquisas publicadas $[2,21,25]$.

É evidente a necessidade de pesquisas sobre a tríplice associação: idoso, saúde mental e risco de quedas, que são condições frequentes em portadores de doenças crônicas. A depressão compromete o desempenho funcional e torna os idosos mais dependentes e vulneráveis às comorbidades, agravando esse problema de saúde pública. O transtorno depressivo pode ser tratado clinicamente e não deve ser encarado como uma consequência natural do envelhecimento [16,26,27,28]. A sugestão é traçar estratégias de rastreamento sistemático, priorizando a que permeiam a vida dos idosos.

Tendo em vista a responsabilidade ética deste estudo, os resultados do mesmo foram apresentados formalmente aos gestores do CCI da Prefeitura Municipal de Vitória da Conquista. Foram demonstrados os percentuais observados, esclarecendo sobre os riscos de queda e depressão, de forma a servir de subsídio aos gestores e equipe multiprofissional para a implantação de medidas de controle dos riscos. A Política Nacional de Saúde do Idoso assegura à pessoa idosa, ações que promovam o envelhecimento saudável, a manutenção da capacidade funcional, o apoio ao desenvolvimento de cuidados específicos e apoio a realização de pesquisas [7,29]. A capacidade da EDG-15 para rastrear a depressão, associada ao autorrelato de quedas, sugere uma relação preditiva que requer novas pesquisas em busca do conhecimento mais profundo sobre os fatores determinantes entre essas variáveis.

A limitação desta pesquisa consistiu no pequeno tamanho amostral e no desenho transversal do estudo, que não permite o estabelecimento das relações de causa e efeito. Entretanto, vale destacar a importância destes resultados para alertar que a depressão em idosos por vezes passa despercebida. As escalas de rastreamento podem ser inseridas na rotina assistencial de médicos e demais profissionais de saúde que sejam devidamente capacitados para identificar traços depressivos com instrumentos simples, valorizando a promoção da saúde mental e a prevenção da doença.

\section{REFERÊNCIAS}

1. Gonçalves VC, Andrade KL. Prevalência de depressão em idosos atendidos em ambulatório de geriatria da região nordeste do Brasil (São Luís-MA). Rev Bras Geriatr Gerontol. 2010;13(2):289-300. http://dx.doi.org/10.1590/S1809-98232010000200013

2. Gonçalves D, Altermann C, Vieira A, Machado AP, Fernandes R, Oliveira A, Mello-Carpes PB. Avaliação das funções cognitivas, qualidade de sono, tempo de reação e risco de quedas em idosos institucionalizados. Estud Interdiscipl Envelhec. 2014;19(1):95-108.

3. Instituto Nacional de Geografia e Estatística (IBGE). Censo demográfico 2010 [Internet]. Brasília; 2010. [cited 201323 Dez]. Available at: http://censo2010.ibge.gov.br

4. Leal MCC, Apostolo JLA, Mendes AMOC, Marques APO. Prevalência de sintomatologia depressiva e fatores associados entre idosos institucionalizados. Acta Paul Enferm. 2014;27(3):208-14. http://dx.doi.org/10.1590/1982-0194201400036

5. Menezes RL, Bachion MM. Estudo da presença de fatores de riscos intrínsecos para quedas, em idosos institucionalizados. Cien Saude Colet. 2008;13(4):1209-18. http://dx.doi.org/10.1590/S1413-81232008000400017

6. Marques WV, Cruz VA, Rego J, Silva NA. Influência da capacidade funcional no risco de quedas em adultos com artrite reumatóide. Rev Bras Reumatol. 2014;54(5):404-8. http://dx.doi.org/10.1016/j.rbr.2014.03.019

7. Rodrigues RAP, Kusumota L, Marques S, Fabrício SCC, Rosset-Cruz I, Lange C. Política nacional de atenção ao idoso e a contribuição da enfermagem. Texto Contex Enferm. 2007;16(3): 536-45. http://dx.doi.org/10.1590/S0104-07072007000300021

8. Biderman A, Cwikel J, Fried AV, Galinsky D. Depression and falls among community dwelling elderly people: a search for common risk factors. J Epidemiol Community Health. 2002;56(8):631-6. http://dx.doi.org/10.1136/jech.56.8.631

9. Valcarenghi RV, Santos SSC, Barlem ELD, Pelzer MT, Gomes GC, Lange C. Alterações na funcionalidade/cognição e depressão em idosos institucionalizados que sofreram quedas. Acta Paulista Enferm. 2011;24(6):828-33. http://dx.doi.org/10.1590/S0103-21002011000600017

10. Alvarenga MRM, Oliveira MAC, Faccenda O. Sintomas depressivos em idosos: análise dos itens da Escala de Depressão Geriátrica. Acta Paul Enferm. 2012;25(4):497-503. http://dx.doi.org/10.1590/S0103-21002012000400003

11. Damasio BF. Uso da análise fatorial exploratória em psicologia. Aval Psicol. 2012;11(2):213-28.

12. Gai J, Gomes L, Nobrega OT, Rodrigues MP. Fatores associados a quedas em mulheres idosas residentes na comunidade. Rev Assoc Med Bras. 2010;56(3):327-32. http://dx.doi.org/10.1590/S0104-42302010000300019

13. Borges DT, Dalmolin BM. Depressão em idosos de uma comunidade assistida pela Estratégia de Saúde da Família em Passo Fundo, RS. Rev Bras Med Fam Comun. 2012;7(23):75-82. http://dx.doi.org/10.5712/rbmfc7(23)381

14. Bandeira CB. Prevalência de depressão em idosos Institucionalizados. [dissertação]. [Fortaleza]: Universidade de Fortaleza-CE- UNIFOR; 2012. $60 \mathrm{p}$. 
15. Fraguas Jr. R, Alves TCTF. Depressão no Hospital Geral: estudo de 136 casos. Rev. Assoc. Med. Bras. 2002;48(3): 225-30. http://dx.doi. org/10.1590/S0104-42302002000300035

16. Santos SSC, Tier CG, Silva BT, Barlem ELD, Felicianni AM, Valcarenghi FV. Diagnósticos e intervenções de enfermagem para idosos deprimidos e residentes em uma instituição de longa permanência (ILP). Rev Eletrônica Enfermeria. 2010;20(01):2-14.

17. Vaz, SFA, Gaspar NMS. Depressão em idosos institucionalizados no distrito de Bragança. Rev Enf Ref . 2011;03(4):49-58. http://dx.doi. org/10.12707/RIII1124

18. Pelegrin AKAP, Araujo JÁ, Costa LC, Cyrillo RMZ. Rosset I. Idosos de uma Instituição de Longa Permanência de Ribeirão Preto: níveis de capacidade funcional. Arq Ciênc Saúde. 2008;15(4):182-8.

19. Dancey CP, Reidy J. Estatística sem matemática para a psicologia. 5ed. Porto Alegre: Penso, 2013.

20. Almeida EAD, Madeira GD, Arantes PMM, Alencar ME. Comparação da qualidade de vida entre idosos que participam e idosos que não participam de grupos de convivência na cidade de Itabira-MG. Rev Bras Geriat Geront. 2010;13(3):435-43. http://dx.doi.org/10.1590/ S1809-98232010000300010

21. Pinho MX, Custódio O, Makdisse M, Carvalho ACC. Confiabilidade e validade da escala de depressão geriátrica em idosos com doença arterial coronariana. Arq Bras Cardiol. 2010;94(5):570-9. http://dx.doi.org/10.1590/S0066-782X2010005000032

22. Paradela EMP, Lourenço RA, Veras RP. Validação da escala de depressão geriátrica em um ambulatório geral. Rev Saúde Pública. 2005;39(6):918-23. http://dx.doi.org/10.1590/S0034-89102005000600008

23. Almeida OP, Almeida SA. Confiabilidade da versão brasileira da Escala de Depressão Geriátrica (GDS) versão reduzida. Arq Neuropsiquiatr. 1999; 57(2B):421-6. http://dx.doi.org/10.1590/S0004-282X1999000300013

24. Oliveira MA, Batistoni SST, Melo RC, Yassuda MS, Domingues MA, Lopes A, Cachioni M. Questionário de Expressão Emocional aplicado a idosos: indicadores psicométricos e relações com variáveis sociodemográficas e afetivas. Rev Tem Kairós Gerontol. 2012;15(7):89-106.

25. Minicuci N, Maggi S, Pavan M, Enzi G, Crepaldi G. Prevalence rate and correlates of depressive symptoms in older individuals: the Veneto Study. Journals of Gerontology. Series A: Biological and Medical Sciences. 2002; 57(3):155-61. http://dx.doi.org/10.1093/ gerona/57.3.M155

26. Leite VMM, Carvalho EMF, Barreto KML, Falcão IV. Depressão e envelhecimento: estudo com participantes do Programa Universidade Aberto à Terceira Idade. Rev Bras Saúde Mater Infant. 2006;6(1):31-8. http://dx.doi.org/10.1590/S1519-38292006000100004

27. Angelim RM. Incidência de sintomas depressivos em idosos portadores e não portadores de doenças crônicas: um estudo na rede de Atenção Básica à Saúde de Campina Grande.[tese]. [Campina Grande]: Universidade Estadual da Paraíba; 2012. 31 p.

28. Prata, HL, Alves Jr. ED, Paula FL, Fereira SM. Envelhecimento, depressão e quedas: um estudo com os participantes do Projeto Prev-Quedas. Rev Fisioter Mov. 2011;24(3):437-43.

29. Brasil, Senado Federal Brasileiro-SFB. Lei Ordinária 1994. Brasília; 1994. [cited 06 nov. 2014]. Available at: http://www.senado.gov.br 